\title{
New developments in active and passive 3D image sensing, visualization, and processing
}

\author{
Bahram Javidi $^{1}$, Enrique Tajahuerce ${ }^{2}$, Manuel Martínez-Corral ${ }^{3}$, \\ Thomas J. Naughton ${ }^{4}$, and Yann Frauel ${ }^{5}$ \\ ${ }^{1}$ Electrical and Computer Engineering Department, University of Connecticut, \\ Storrs, CT 06269-2157, USA. \\ ${ }^{2}$ Departament de Ciències Experimentals, Universitat Jaume I, 12080 Castellón, Spain. \\ ${ }^{3}$ Departamento de Óptica, Universidad de Valencia, 46100 Burjassot, Spain. \\ ${ }^{4}$ Department of Computer Science, National University of Ireland/Maynooth, \\ Maynooth, County Kildare, Ireland. \\ ${ }^{5}$ IIMAS, Universidad Nacional Autónoma de México, 01000 México, D.F., México.
}

\begin{abstract}
We review two different techniques for visualization and processing of three-dimensional (3D) objects based on passive and active optical sensing. First, we describe the basis of a passive-sensing technique based on integral imaging. Also, we show that it is possible to improve the depth of field of this method by using amplitude-modulated microlens arrays. Second, we describe an active-sensing technique based on digital holography. Finally, we apply both techniques to develop 3D image processing applications. In particular, we design two different 3D pattern recognition techniques. Both of them are based in storing the 3D data in two-dimensional (2D) form. In this way, it is possible to recognize 3D objects by performing 2D correlations or applying neural network techniques. Experimental results are presented.
\end{abstract}

Keywords: Three-dimensional imaging, three-dimensional object recognition, integral imaging, digital holography

\section{INTRODUCTION}

Optoelectronic techniques for 3D image sensing, visualization and processing have been the subject of extensive research in the last years. ${ }^{1-31}$ Advances in these fields have been possible in part due to tremendous development in computer technology, light sources, imaging devices, and spatial and temporal light modulators. In principle, the procedures to obtain information about the structure of 3D objects can be divided into two categories, passive and active sensing techniques.

In the first category, based on passive sensing, we find methods such as triangulation or more recent ones as the so-called integral imaging. In particular, integral imaging is a 3D imaging technique specially suited to work with incoherent light, and provides with autostereoscopic images without the help of any special glasses. ${ }^{7-19}$ In an integralimaging system, an array of microlenses generates a collection of 2D elemental images of the D object onto a sensor such as a CCD. Each elemental image has a different perspective of the 3D object. In the reconstruction stage, the recorded images are displayed by an optical device, such as a LCD monitor, placed in front of another microlens array, providing the observer with a reconstructed 3D image with full paralax.

One of the main challenges in integral imaging is to overcome its limited depth of field. However, new methods to significantly extend the depth of field have been proposed. ${ }^{17-19}$ These techniques take into account the sensor resolution constraints, and are based on the combined benefits of a proper amplitude modulation of the microlenses, and the application of deconvolution tools.

Based on integral imaging, a 3D optical pattern recognition technique has been developed already. ${ }^{9}$ It uses the arrays of 2D elemental images generated by the microlens array to perform 2D correlations. In this way, it is possible to detect the presence of the reference 3D object and to measure out-of-plane rotation in 3D input scenes.

Unmanned/Unattended Sensors and Sensors Networks II,

Edited by Edward M. Carapezza, Proc. of SPIE Vol. 5986

598601, (2005) · doi: 10.1117/12.633672

Proc. of SPIE Vol. 5986 598601-1 
In the second category, based on active sensing, we find techniques such as optical coherence tomography and holography. Specifically, digital holography is a variant of regular optical holography that provides holograms in digital form by recording interference patterns with a CCD. ${ }^{20}$ It avoids analogue recording and corresponding chemical development, and allows one to record, process, and transmit phase and amplitude information digitally. These techniques have been extended to security applications, such as recognition, encryption, and authentication of 3D information. ${ }^{21-33}$

A efficient approach to record the digital hologram of a 3D object is to use phase shifting interferometers based on retarder plates. In one approach for 3D object recognition, the complex information in the digital hologram is used directly to detect the presence of a 3D reference object inside a 3D input scene with high discrimination. ${ }^{22}$ In a second approach, the method is extended to achieve shift invariance along the optical axis. ${ }^{23}$ Furthermore, nonlinear composite correlation filters have been used to achieve distortion tolerance, taking advantage of the properties of holograms to make the composite filter from one single hologram. ${ }^{24}$ Also, a two-layer neural network for processing of 3D images that are obtained by digital holography has been developed. ${ }^{25}$ The network is trained with a real 3D object to compute the weights of the layers. In this way, we recognize 3D objects with severe out-of-plane rotation. A comparison between passive ranging integral imaging and active imaging digital holography for 3D object recognition has been done. ${ }^{11}$

Besides pattern recognition, other optoelectronic 3D image processing operations based in the above methods are also possible. In this way, techniques to encrypt 3D information have been developed by using random phase codes when recording the digital hologram. ${ }^{22}$ After digital decryption with the proper key, images of the 3D object with different perspectives and focused at different planes can be generated digitally in a remote site. A technique to reconstruct optically a 3D object has also been designed by using a liquid-crystal spatial light modulator to codify the phase information of the digital hologram. ${ }^{26}$ Furthermore, application of compression techniques to digital holograms allow one the efficient transmission of 3D images. ${ }^{28,29}$ Several lossy methods are used based on subsampling, quantization, bit packing operation, and discrete Fourier transformation. Compression losses in the reconstructed objects were quantified and a speedup metric has been designed.

In his contribution, we briefly review some of the previous techniques for visualization and processing of threedimensional (3D) objects. First, we describe the basis of integral imaging, a passive-sensing technique. Then, we show that it is possible to improve the depth of field of this method by using amplitude-modulated microlens arrays. Second, we describe the fundamentals of digital holography as an active-sensing technique. Finally, we apply both techniques to develop 3D image processing applications. Specifically, we explain two different 3D pattern recognition techniques. Both of them are based in storing the 3D data in two-dimensional (2D) form. In this way, it is possible to recognize 3D objects by just performing 2D correlations or applying neural network techniques. Experimental results are presented.

\section{BASIC THEORY OF INTEGRAL IMAGING}

In an integral-imaging system, a two-dimensional 2D array of 2D elemental images of a given surface 3D object is generated by a microlens array and recorded on a CCD. Each elemental image shows a different perspective of the 3D object. To reconstruct the 3D image, the recorded 2D elemental images are displayed by an optical device, such as a LCD, in front of another microlens array. ${ }^{7}$ Figures 1 (a) and (b) show a system for 3D object recording and another for

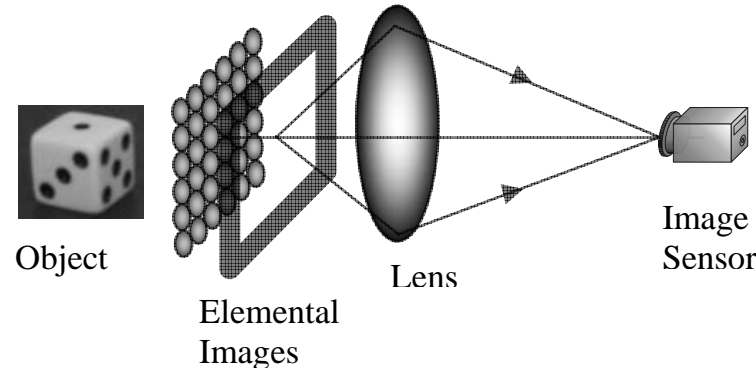

(a)

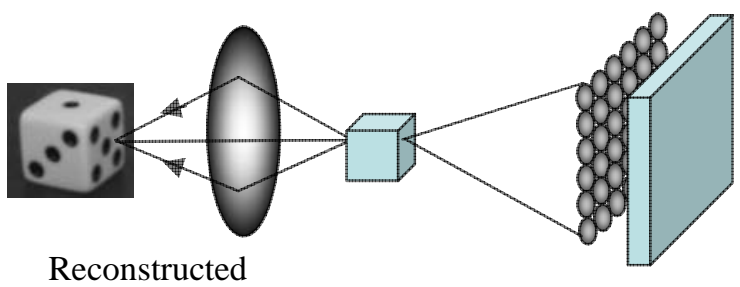

Object
Lens Array
SL

(b)

Figure 1. An integral imaging system; (a) sensing system and (b) reconstruction system. SLM denotes spatial light modulator. 
reconstruction based on integral imaging.

One problem of integral imaging systems is the limited depth of field. Since only a single plane is used to capture the images, it is not possible for all objects in a 3D scene to be in focus. Then blurred images of objects that are out of focus are obtained. To display a clear integral image of a 3D object it is essential to capture sharp 2D elemental images of it. For this reason the bottleneck of the depth of field in integral imaging is the limited depth of field of the microlens array used in the pickup stage.

\section{INTEGRAL IMAGING WITH IMPROVED DEPTH OF FIELD}

New methods to significantly extend the depth of field have been proposed. The technique takes into account the sensor resolution constraints and is based on the combined benefits of a proper amplitude modulation of the microlenses and the application of deconvolution tools. ${ }^{17-19}$ Here we describe only an amplitude modulation technique.

The easiest way to improve the depth of field of the capture setup is by reducing the NA of the microlenses, but such an improvement is accompanied by a proportional deterioration of lateral resolution. This problem can be overcome by use of amplitude-modulation techniques. ${ }^{18}$ The method consists on obscuring the central part of each microlens. Such an obscuration allows the secondary Huygens wavelets proceeding from the outer part of the lenses to interfere constructively in an enlarged axial range. Then, by simply placing an opaque circular mask just behind each microlens, one can increase the focal depth of the microlens array. It is known that the higher the value of the obscuration ratio, the broader the axial intensity spot but, also, the smaller the light efficiency of the system. For this reason the obscuration ratio is selected in such a way that it has a light efficiency of $50 \%$ and doubles the depth of focus of the system.

In Fig. 2 and 3 we show the results of a computer simulation. The diameter of the lenses is $2 \mathrm{~mm}$, the focal length $5 \mathrm{~mm}$, the wavelength is $0.5 \mu \mathrm{m}$, and the distance from a reference plane in the input 3D scene (located ap-

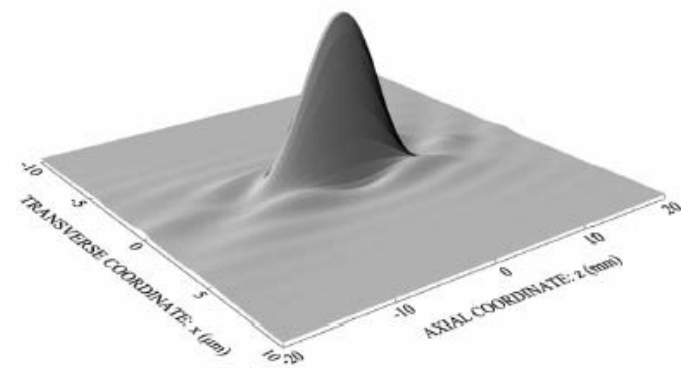

(a)

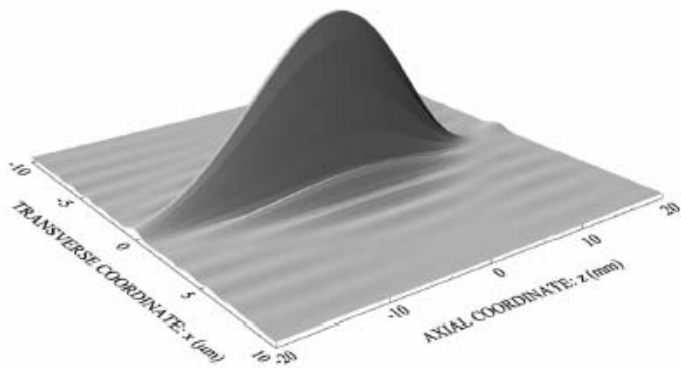

(b)

Figure 2. 3D plot of the meridian section of the impulse response for: (a) a conventional microlens and (b) an amplitude-modulated microlens.

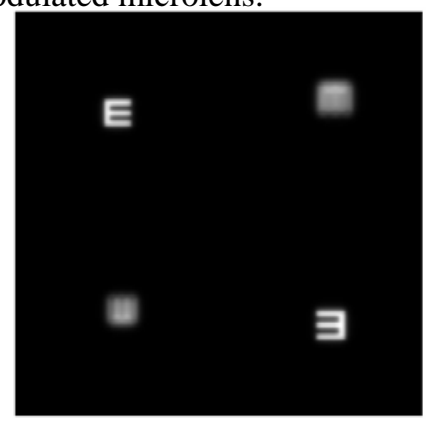

(a)

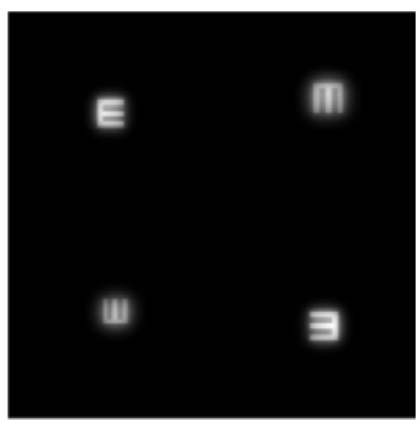

(b)

Figure 3. One of the elemental images captured with: (a) a conventional microlens array and (b) an amplitude-modulated microlens array. The 3D scene object contains tumbling charts located at different distances. 
proximately in the center of the scene) and the microlens array is $100 \mathrm{~mm}$. Figure 2(a) shows a 3D plot of the meridian section of the impulse response of a microlens, while Fig. 2(b) shows the 3D plot of the same function for the case of the amplitude-modulated microlens. Figure 3(a) shows one of the elemental images obtained with a conventional microlens array. The input scene is a set of tumbling charts with different orientations and located at different distances from the array. It is clearly noticeable the limited depth of filed of the system. Figure 3(b) shows the elemental image obtained with the amplitude-modulated microlens array. Improvement in the depth of field is clearly seen.

\section{3D OBJECT RECOGNITION BASED ON INTEGRAL IMAGING}

A joint transform correlation technique can be used to recognize 3D objects starting from an integral imaging recording system. ${ }^{9}$ The correlation between the input 3D scene and the reference 3D object can be calculated optically or numerically. We present here the combination of an optical and a numerical method to obtain the correlation signal. First, two arrays of elemental images, one for the reference scene and another for the input object are recorded optically with an integral imaging system. In Fig. 4 we show some elemental images obtained in this way for the reference and the input object. The joint power spectrum of these signals is also obtained optically by locating the array of elemental images of the input 3D object and reference object together in a photographic film at the input plane of an optical Fourier trans-

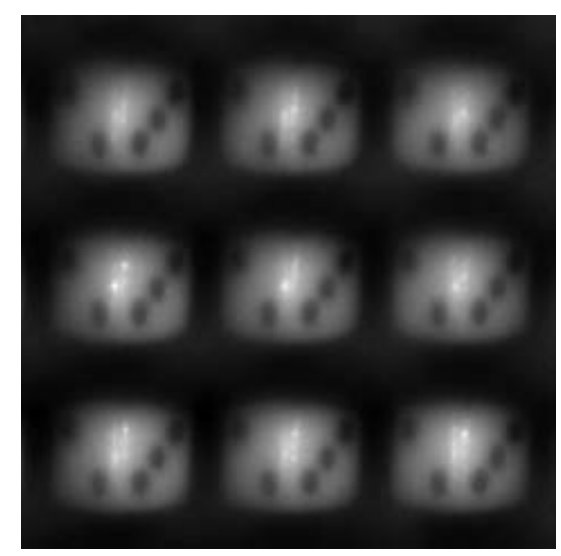

(a)

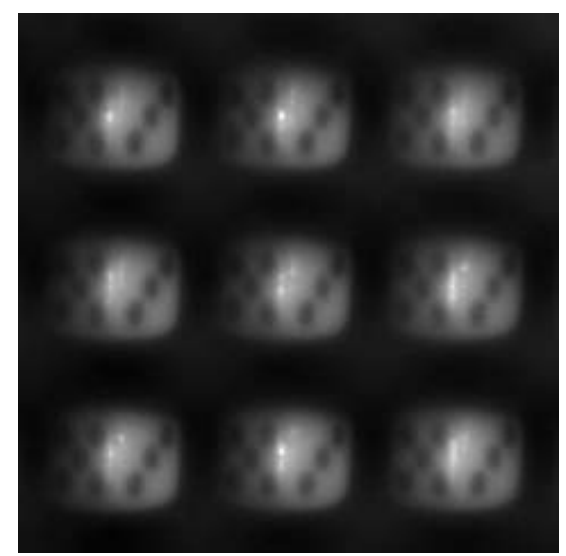

(b)

Figure 4. Part of elemental images of (a) the reference and (b) the input with similar faces of the reference.

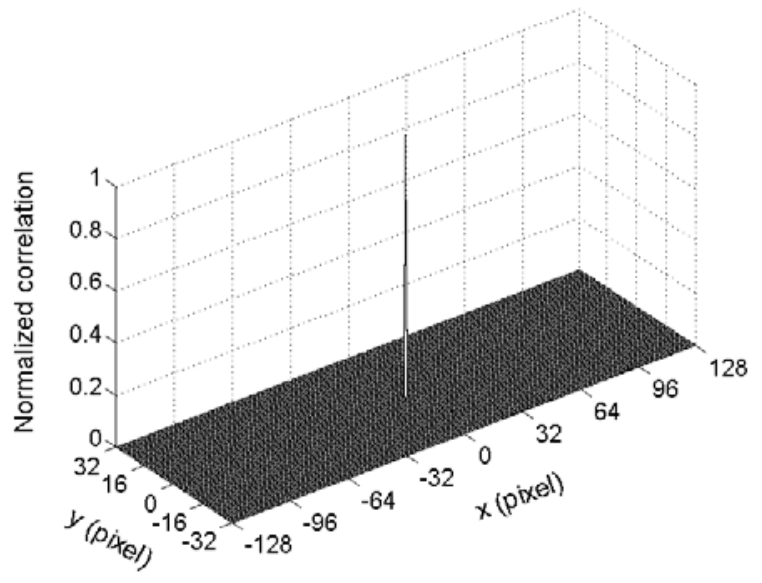

(a)

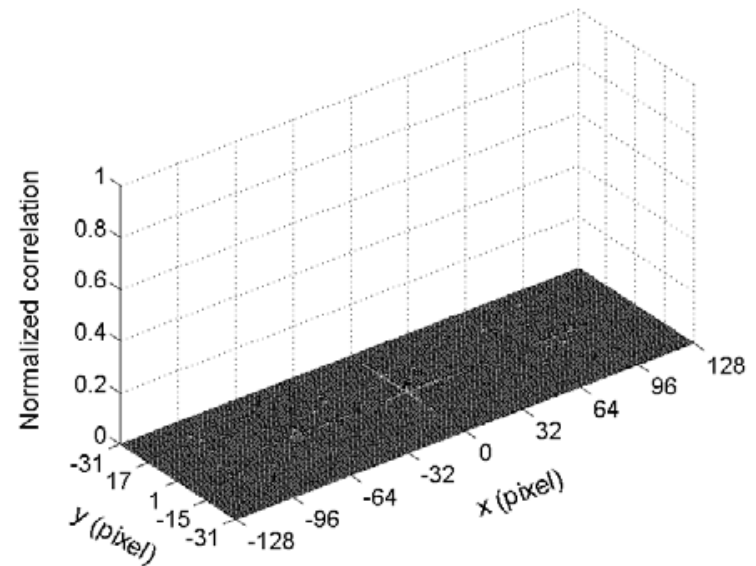

(b)

Figure 5. Correlation results: (a) autocorrelation and (b) crosscorrelation with similar face. 


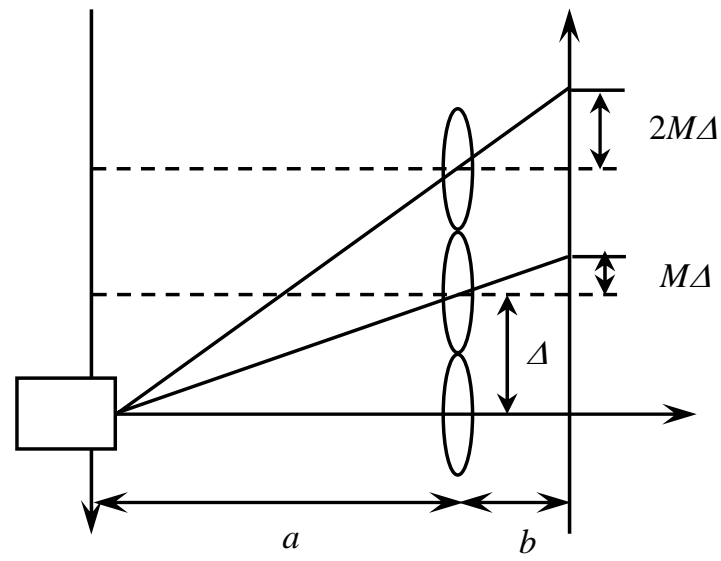

(a)

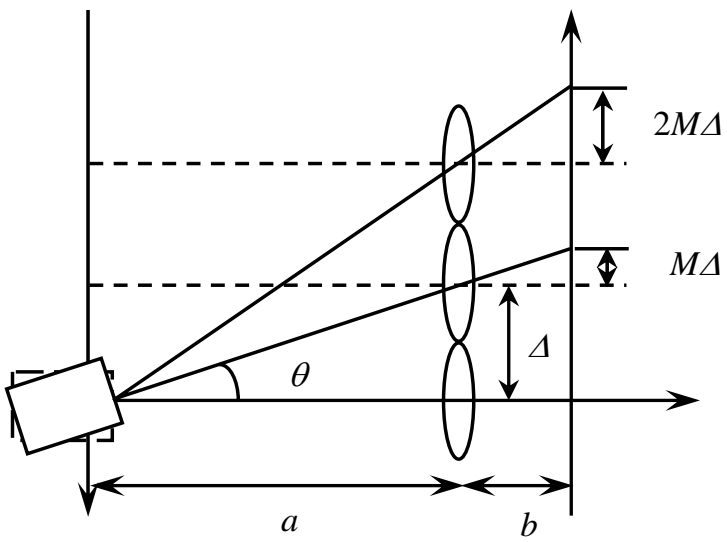

(b)

Figure 6 .Spatial shift of elemental images when the 3D object is rotated.

former. Finally, we evaluate the Fourier transform of the joint power spectrum in a computer, in order to obtain the correlation of the object and the reference. Figures 5(a) and (b) show the autocorrelation and crosscorrelation of the input scene and the reference 3D object. We can see that the autocorrelation has a sharp peak, however the peak could not be seen in the crosscorrelation. The crosscorrelation signal is 14.6 times smaller than the autocorrelation signal. Here the joint power spectrum is binarized to enhance the correlation signal.

This system can also recognize a slightly rotated object and can evaluate the rotation angle. Figure 6 depicts the projection of a 3D input into elemental 2D images. Each lens sees the input 3D object from different angles. When the input 3D object is rotated by a given angle, the elemental images are shifted. This spatial shift in the elemental images results in the shift of the corresponding correlation peak when we compare the set of elemental images before and after the object rotation. Figure 7 shows crosscorrelation profiles between the reference and the rotated reference with rotation angles of 0.26 and $0.78^{\circ}$. There are several sharp peaks in both plots. The highest peak located at 0 represents the autocorrelation due to the similar structure of the die in both cases, with and without rotation. The second highest peak gives information about the rotation angle. When the rotation angle changes, the crosscorrelation peak also shifts as is shown in Figs. 7(a) and (b).

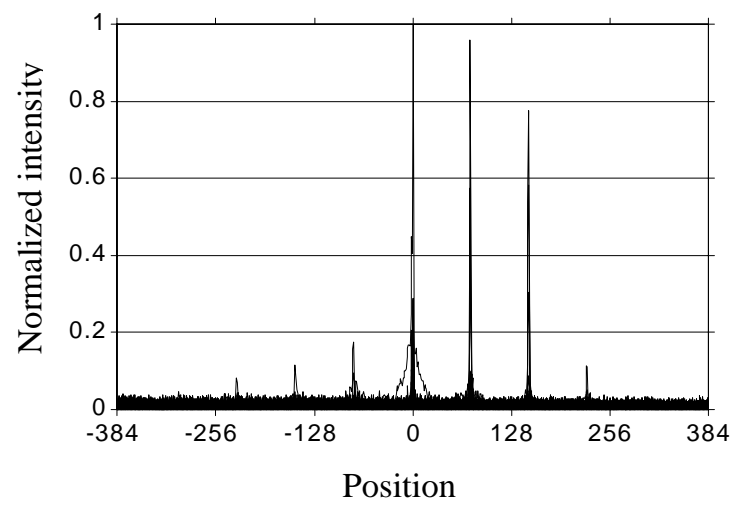

(a)

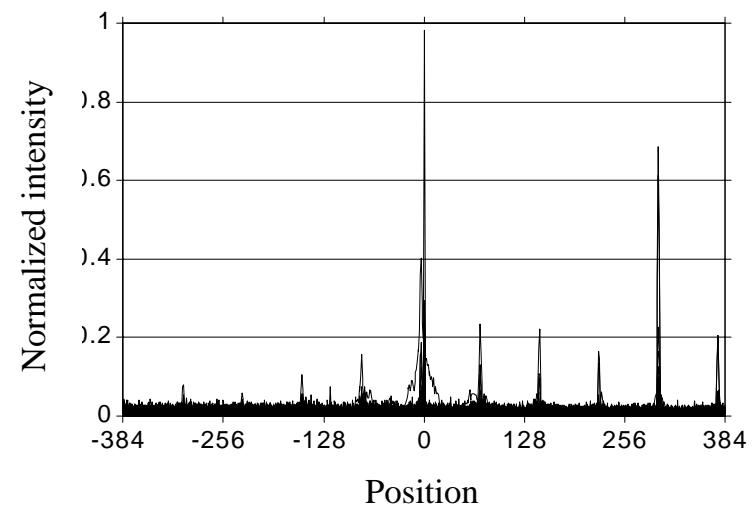

(b)

Figure 7. Correlation profile when the die is rotated by an angle of (a) $0.26^{\circ}$ and (b) $0.78^{\circ}$. 


\section{PHASE SHIFTING DIGITAL HOLOGRAPHY}

It is well known that holography can record 3D information of an object because it allows one to record both the amplitude and phase distribution of the light diffracted by the object. Holograms have been recorded in digital format by using an electronic image sensor instead of a photographic plate. In the same way than conventional hologram reconstructs the 0 -th order, the object wave and the conjugate of the object wave, conventional digital holograms have also the same problem. By use of phase shifting digital holography, it is possible to reconstruct the object wave only or its conjugate wave separately. ${ }^{20}$ Phase shifting digital holography has been used in many applications such as measurement, display, and security systems.

Figure 8 shows a typical configuration of a system for phase-shifting digital holography with retarder plates. ${ }^{21} \mathrm{~A}$ coherent light beam is divided into the object and the reference beam. The object beam illuminates a 3D object and the diffracted light propagates through free space. The object beam is interfered with the reference beam. The interference pattern is recorded as a digital hologram by an image sensor. In the four-step phase shifting technique, the phase of the reference beam is modulated by $0, \pi / 2, \pi$, and $3 \pi / 2$. This is done by phase retarders such as wave plates, liquid crystal modulators, or other electro-optic modulators. If we denote the object and the reference amplitude distributions at the sensor plane by $U_{o}$ and $U_{r}$, respectively, the irradiance distribution of the interference pattern is written as

$$
I_{n}(x, y)=\left|U_{o}+U_{r} \exp \left(j n \frac{\pi}{2}\right)\right|^{2}
$$

with

$$
U_{o}(x, y)=A_{o}(x, y) \exp \left(-j \phi_{o}(x, y)\right)
$$

and

$$
U_{r}(x, y)=A_{r}(x, y) \exp \left(-j \phi_{r}(x, y)\right),
$$

where $n=0,1,2,3$. By using four interference patterns, the amplitude and the phase of the object beam can be described as

$$
A_{o}(x, y)=\frac{\sqrt{\left(I_{0}-I_{2}\right)^{2}+\left(I_{1}-I_{3}\right)^{2}}}{2 A_{r}}
$$

and the phase as

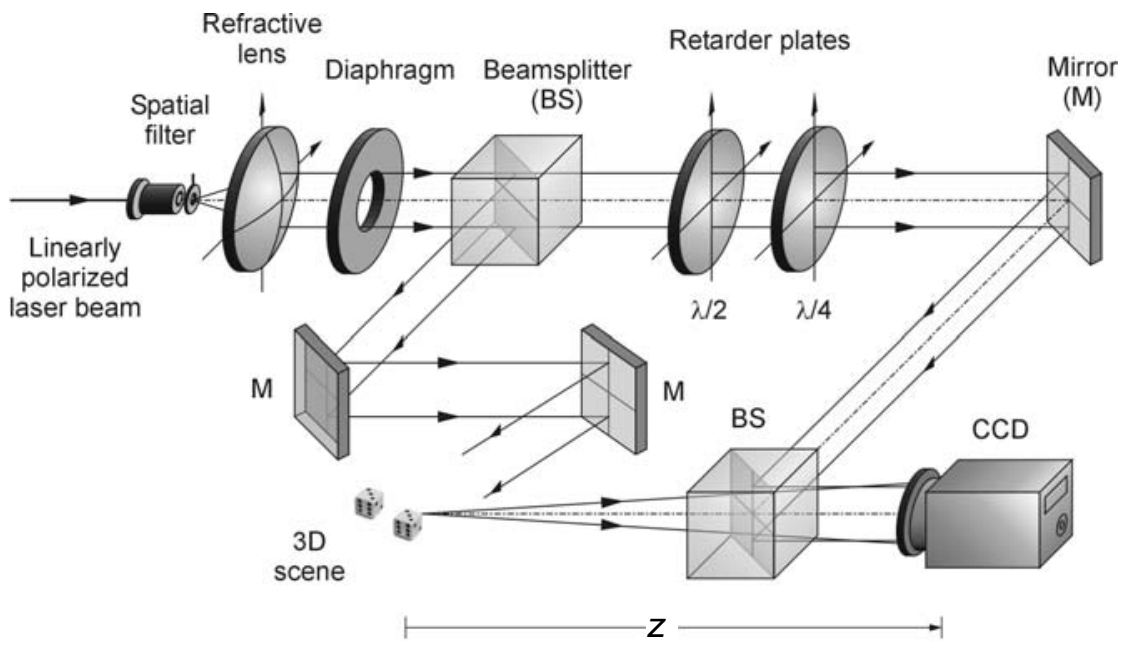

Figure 8. Optical configuration for a four-step phase shifting digital holography system. SP, spatial filter; L, lens; A, aperture; BS’s, beamsplitter; M’s, mirror; RP’s, phase retarder plate. 


$$
\phi_{o}(x, y)=\phi_{r}(x, y)+\tan ^{-1}\left(\frac{I_{1}-I_{3}}{I_{0}-I_{2}}\right) .
$$

When a plane wave is used as reference beam, it is easy to calculate Eqs. (4) and (5). The calculation is implemented by using local information of irradiance at each pixel.

After calculation of the optical field, we can reconstruct the 3D object in a computer or an optical system. Here we present a numerical reconstruction method. Fresnel propagation with a propagation distance $z$ can be evaluated by using the following equation:

$$
U(u, v, z)=\frac{\exp (j k z)}{j \lambda z} \iint U_{o}(x, y) \exp \left[-j \frac{k}{2 z}\left\{(u-x)^{2}+(v-y)^{2}\right\}\right] d x d y .
$$

Because of the sampling theory, we need to change the calculation method according to the range of distances z. For short distances, the Fresnel propagation is better calculated by convolution in the following way

$$
U(u, v, z)=\frac{\exp (j k z)}{j \lambda z} \mathrm{FT}^{-1}\left[\mathrm{FT}\left[U_{o}(x, y)\right] \cdot \mathrm{FT}\left[\exp \left\{-j \frac{k}{2 z}\left(x^{2}+y^{2}\right)\right\}\right],\right.
$$

where $\mathrm{FT}^{-1}[\bullet]$ and $\mathrm{FT}[\bullet]$ denote the inverse Fourier transform and Fourier transform, respectively. However, for long distances, the reconstruction can be better calculated with only one Fourier transformation:

$U(u, v, z)=\frac{\exp (j k z)}{j \lambda z} \exp \left\{-j \frac{k}{2 z}\left(u^{2}+v^{2}\right)\right\} \mathrm{FT}\left[U_{o}(x, y) \exp \left\{-j \frac{k}{2 z}\left(x^{2}+y^{2}\right)\right\}\right]$.

In Fig. 9, we show an image of a 3D object obtained by reconstructing numerically a digital hologram obtained with an optical system similar to that in Fig. 8. The sensor is a CCD with pixel size equal to $9 \mu \mathrm{m} \times 9 \mu \mathrm{m}$. The object is a 3D die with dimensions $4.6 \times 4.6 \times 4.6 \mathrm{~mm}$ and is located at a distance $d=570 \mathrm{~mm}$ from the CCD.

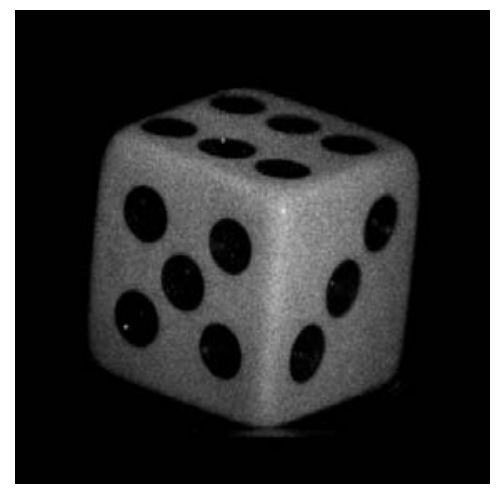

Figure 9. Image of a 3D object obtained by phase-shift interferometry with the optical system in Fig. 8.

\section{3D RECOGNITION BASED ON DIGITAL HOLOGRAPHY}

We have developed a 3D object recognition system based on phase shifting digital holography. ${ }^{22,23}$ In our first approach, we calculate the crosscorrelation between the complex fields of the input scene and the reference 3D object measured by digital holography. The experimental results show that the correlation is highly sensitive to displacements of the 3D objects because of the speckle noise caused by the rough surfaces of the objects. In a practical application, a more flexible technique is required.

In our second approach, we develop a 3D object recognition system by using the reconstructed irradiance distributions from the digital holograms. The phase information is dropped and, thus, the correlation results becomes more 


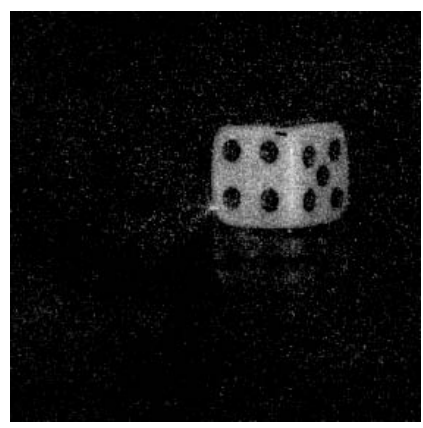

(a)

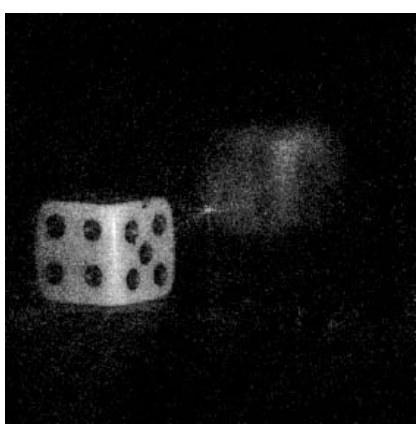

(b)

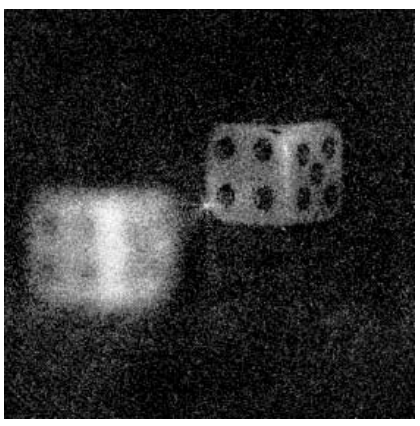

(c)

Figure 10. (a) Reconstructed image of the reference at $345 \mathrm{~mm}$ from the image sensor. Reconstructed image of the input scene at (b) $315 \mathrm{~mm}$ and (c) $345 \mathrm{~mm}$ from the image sensor.

robust. However it takes a long time to calculate 3D correlation by use of reconstructed 3D object. Therefore we use a reconstructed 2D image of the reference 3D object at an appropriate plane. A series of 2D correlation between the reference and a series of reconstructed input object is calculated.

In our experiment we use the same dice, with dimensions $4.6 \mathrm{~mm} \times 4.6 \mathrm{~mm} \times 4.6 \mathrm{~mm}$, for the 3D input scene and for the 3D reference. The 3D reference is a die located at $345 \mathrm{~mm}$. The 3D input scene contains two dice located at 315 $\mathrm{mm}$ and $345 \mathrm{~mm}$. The pixel size of the image sensor is $9 \mu \mathrm{m} \times 9 \mu \mathrm{m}$. Figure 10 shows several numerical reconstruction of the die obtained by digital holography with a system similar to that in Fig. 8. Figure 10(a) is the reconstructed image of the reference 3D object at a propagation distance of $345 \mathrm{~mm}$ from the image sensor. We evaluate the reconstructed image of the 3D input scene at various propagation distances z. In Figs. 7(b) and (c) we show two examples at propagation distances of $315 \mathrm{~mm}$ and $345 \mathrm{~mm}$ from the image sensor. Then, we calculate 2D correlations between the reconstructed image of the reference and a series of reconstructed images of the input. Figure 11 shows the correlation peak profile. We can see that there are two local maxima. One of the objects is located at the same position as the reference. The second one is located at a 3D coordinate with respect to the reference object given by $(-7.7,-3.4,-30) \mathrm{mm}$. The peak of the second one is smaller than the first one. This is caused by the fact that the second object is not identical to the reference and is slightly rotated from the reference.

Because of the nature of holography, it is possible to reconstruct the image of the 3D object with different views by use of a noncentered window in the hologram plane. We can use this property to measure the rotation angle of the input 3D object with respect to the reference. To this end, we calculated reconstructions of a 3D input object located at a distance of $315 \mathrm{~mm}$ for different perspective angles. Figure 12 shows reconstructed images for only three perspective angles: $-0.9^{\circ}, 0^{\circ}$, and 0.9 . We also calculated the correlation between the different reconstructions of the input and one

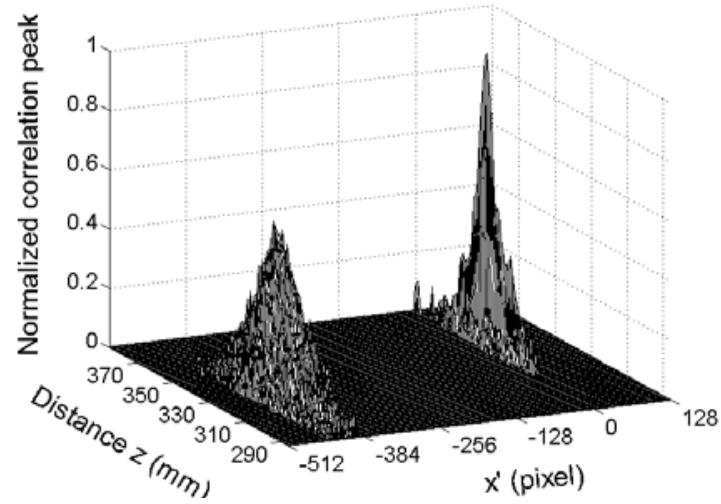

Figure 11. Value of the correlation peak at fixed positions (those of maximum correlation peaks), for input images reconstructed at various propagation distances $\mathrm{z}$. 


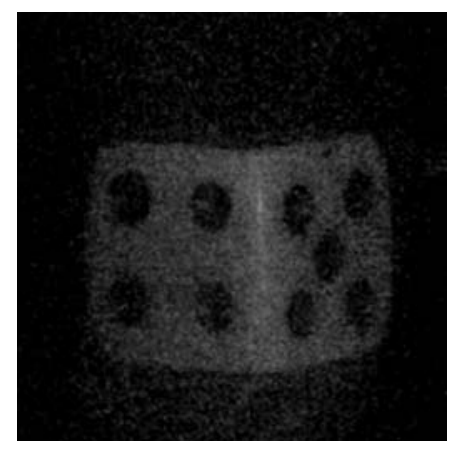

(a)

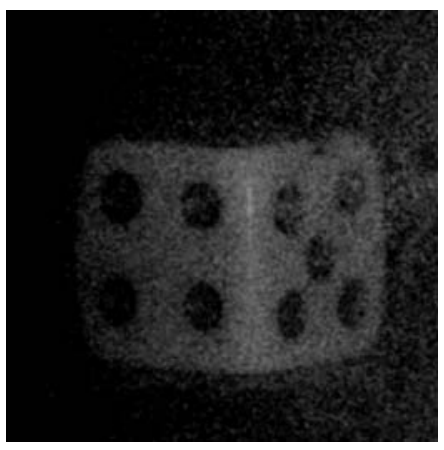

(b)

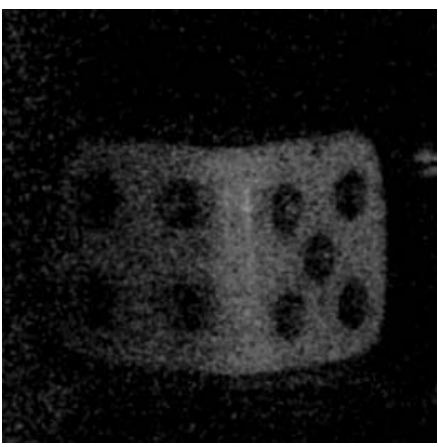

(c)

Figure 12. Reconstructed images at $315 \mathrm{~mm}$ from the image sensor for three different perspective angles: (a) $-0.9^{\circ}$, (b) $0^{\circ}$, and (c) $0.9^{\circ}$.

reconstruction of the 3D reference. The correlation peak for each perspective is shown in Fig. 13. We can see that the correlation peak is obtained at $-0.9^{\circ}\left(-544\right.$ pixels). This means that the input die is rotated by $-0.9^{\circ}$ with respect to the reference die.

As mentioned above, the rotation tolerance is limited to small angles, about $1^{\circ}$, because of the small effective numerical aperture of the lensless system in Fig. 8. We have already showed that it is possible to enhance the rotation tolerance by use of nonlinear composite filters. ${ }^{24}$ The classification capability can also be improved by use of neural networks. ${ }^{25}$

\section{CONCLUSIONS}

We have presented two techniques for recording and visualizing 3D objects by use of passive and active optical sensing. First, to measure 3D object information, we have used integral imaging, as an example of passive sensing technique. We have shown also that it is possible to improve the depth of field of integral imaging systems by amplitude modulation. Second, we have used phase-shifting digital holography, an active sensing technique, to record 3D infor-

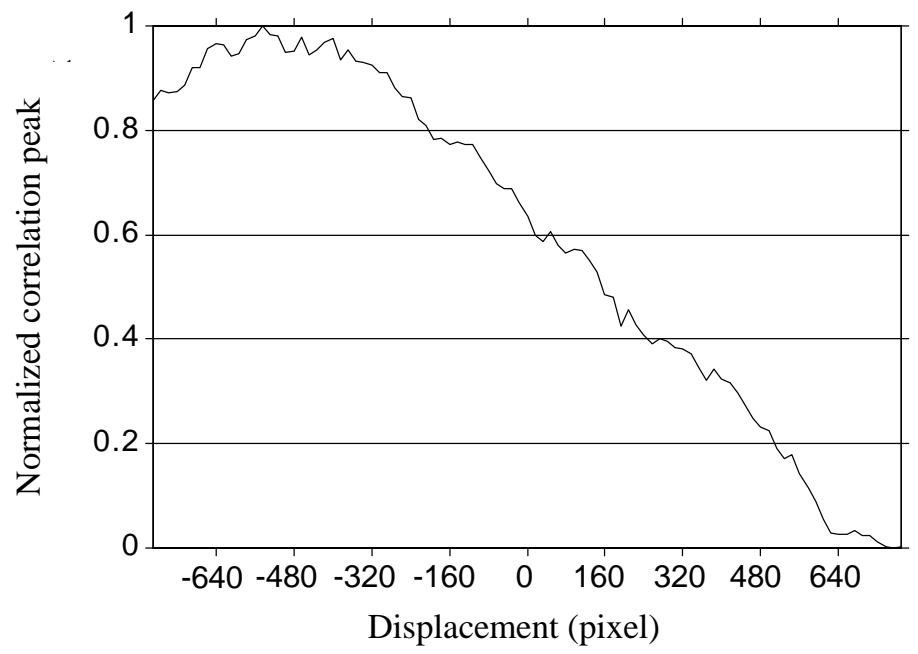

Figure 13. Maximum value of correlation between the reference and the input reconstructed images with different angle of views by use of noncentered windows. 
mation.

Integral imaging has several advantages over phase shifting digital holography as, for example, the fact that incoherent light sources can be used, speckle noise is avoided, and one-shot measurement is possible. However, image quality in integral imaging is inferior to that of phase shifting digital holography. On the other hand, three or four interference patterns are required to calculate the optical complex field with phase shifting digital holography. The sensing and numerical reconstruction in digital holography can be implemented in parallel, thus the system can be faster by making hardware with CMOS technology. Both methods can be active by use of modulated light sources, modulators, and appropriate detection systems.

Because 3D correlations takes a long time in numerical calculation, we have demonstrated the ability to perform 3D recognition experiments by performing 2D correlations. Experimental results show that recognition capability of the present systems is high even when the objects similar to the reference were used. The results also show that rotation of 3D objects can be measured.

We would like to point out that, besides pattern recognition, other optoelectronic 3D image processing operations based in the above methods are also possible. Recently, we have reported techniques to encrypt 3D information, to reconstruct optically a 3D object and to compress digital holograms.

\section{REFERENCES}

[1] T. Okoshi, Three-dimensional Imaging Techniques (Academic Press, New York, 1971).

[2] B. Javidi ed., Image Recognition and Classification: Algorithms, Systems, and Applications (Marcel-Dekker, New York, 2002).

[3] B. Javidi and F. Okano eds, Three Dimensional Television, Video, and Display Technologies (Springer Verlag Berlin, 2002).

[4] A. Pu, R. Denkewalter, and D. Psaltis, "Real-time vehicle navigation using a holographic memory,” Opt. Eng. 36, 2737-2746 (1997).

[5] J. Rosen, “Three-dimensional joint transform correlator,” Appl. Opt. 37, 7538-7544 (1998).

[6] S. Hong and B. Javidi, "Detecting 3D location and shape of noisy distorted 3D objects with Ladar trained optimum nonlinear filters,” Appl. Opt. 43, 324-332 (2004).

[7] F. Okano, J. Arai, H. Hoshino, and I. Yiyama, "Three-dimensional video system based on integral photography," Opt. Eng. 38, 1072-1077 (1999).

[8] H. Arimoto and B. Javidi, "Integral Three-dimensional Imaging with Computed Reconstruction,” Opt. Lett. 26 (2001).

[9] O. Matoba, E. Tajahuerce, and B. Javidi, "Real-time three-dimensional object recognition with multiple perspectives imaging,” Appl. Opt. 40, 3318(2001).

[10] Y. Frauel and B. Javidi, "Digital three-dimensional image correlation by use of computer-reconstructed integral imaging,” Appl. Opt. 41, 5488-5496 (2002).

[11] Y. Frauel, E. Tajahuerce, O. Matoba, A. Castro, and B. Javidi, "Comparison of passive ranging integral imaging and active imaging digital holography for three-dimensional object recognition,” Appl. Opt. 43, 452-462 (2004).

[12] O. Matoba and B. Javidi, “Three-dimensional polarimetric integral imaging,” Opt. Lett. 29, 2375-2377 (2004).

[13] J. S. Jang and B. Javidi, "Three-dimensional TV and display with large depth of focus and improved resolution," Opt. and Phot. News, 36-43, April 2004.

[14] M. Hain, W. von Spiegel, M. Schmiedchen, T. Tschudi, B. Javidi, "3D integral imaging using diffractive Fresnel lens arrays," Opt. Express 13, 315-326 (2005).

[15] A. Stern and B. Javidi, "Information capacity gain by time-division multiplexing in 3D integral imaging," Opt. Lett. 30, 1135-1137 (2005). 
[16] J. S. Jang and B. Javidi, "Very-large scale integral imaging (VLSII) for 3D display," Opt. Eng. 44, 1400-1406 (2005).

[17] R. Martínez-Cuenca, G. Saavedra, M. Martínez-Corral, and B. Javidi, "Enhanced depth of field integral imaging with integral imaging with sensor resolutions constraints," Opt. Express 12, 5237-5242 (2004).

[18] M. Martínez-Corral, B. Javidi, R. Martínez-Cuenca, and G. Saavedra, "Integral imaging with improved depth of field by use of amplitude-modulated microlens array," Appl. Opt. 43, 5806-5813 (2004).

[19] M. Martínez-Corral, B. Javidi, R. Martínez-Cuenca, and G. Saavedra, "Multifacet structure of observed reconstructed integral images," J. Opt. Soc. Am. A 22, 597-603 (2005).

[20] I. Yamaguchi and T. Zhang, "Phase-shifting digital holography,” Opt. Lett. 22, 1268-1270 (1997).

[21] E. Tajahuerce and B. Javidi, "Encrypting three-dimensional information with digital holography,” Appl. Opt. 39, 6595-6601 (2000).

[22] B. Javidi and E. Tajahuerce, “Three-dimensional object recognition using digital holography,” Opt. Lett. 25, 610612 (2000).

[23] E. Tajahuerce, O. Matoba, and B. Javidi, "Shift-invariant three-dimensional object recognition by means of digital holography,” Appl. Opt. 40, 3877 (2001).

[24] Y. Frauel, E. Tajahuerce, M.A. Castro, and B. Javidi, "Distortion-tolerant 3D object recognition using digital holography,” Appl. Opt. 40, 3887-3893 (2001).

[25] Y. Frauel, and B. Javidi, "Neural network for three-dimensional object recognition based on digital holography," Opt. Lett. 26, 1478-1480 (2001).

[26] O. Matoba, T.J. Naughton, Y. Frauel, N. Bertaux, and B. Javidi, "Real-time three-dimensional object reconstruction by use of a phase-encoded digital hologram,” Appl. Opt. 41, 6187-6192 (2002).

[27] S. Shin and B. Javidi, "Three-dimensional object recognition by use of a photorefractive volume holographic processor,” Opt. Lett., 26, 1161-1164 (2001).

[28] T.J. Naughton, Y. Frauel, B. Javidi, and E. Tajahuerce, "Compression of digital holograms for three-dimensional object reconstruction and recognition,” Appl. Opt. 41, 4124-4132 (2002).

[29] T. J. Naughton, J. B. McDonald, and B. Javidi, "Efficient compression of Fresnel Fields for Internet transmission of three-dimensional images,” Appl. Opt. 42, 4758-4764 (2003).

[30] D. Kim and B. Javidi, "Distortion-tolerant 3-D object recognition by using single exposure on-axis digital holography," Opt. Express 12, 5539-5548 (2004).

[31] B. Javidi, I. Moon, S. K. Yeom, and E. Carapezza, "Three-dimensional imaging and recognition of microorganism using single-exposure on-line (SEOL) digital holography," Opt. Express 13, 4492-4506 (2005).

[32] B. Javidi and D. Kim, "3-D object recognition using single exposure on-axis digital holography," Opt. Lett. 30, 236-238 (2005).

[33] B. Javidi, P. Ferraro, S. Hong, and D. Alfieri, "3D Image fusion using multi-wavelengths digital holography, Opt. Lett. 30, 144-146 (2005). 